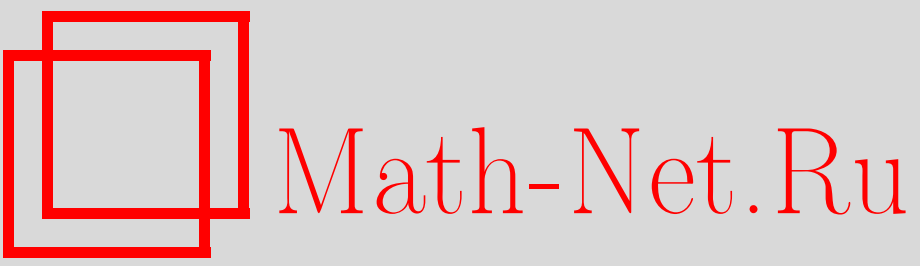

В Санкт-Петербургском математическом обществе. Заседания Общества, УМH, 2004, том 59, выпуск 2, 199-204

DOI: https://doi.org/10.4213/rm731

Использование Общероссийского математического портала Math-Net.Ru подразумевает, что вы прочитали и согласны с пользовательским соглашением

http://www.mathnet.ru/rus/agreement

Параметры загрузки:

IP : 52.23 .180 .231

26 апреля 2023 г., 16:50:50 


\section{В САНКТ-ПЕТЕРБУРГСКОМ МАТЕМАТИЧЕСКОМ ОБЩЕСТВЕ}

\section{Заседания Общества}

Заседание 11 июля 2002 г. Совместное заседание Общества и Общего семинара ПОМИ, посвященное 70-летию М.З.Соломяка (институт Вейцмана, Израиль).

М.3. Соломяк. О спектральных свойствах лапласиана на метрических графах.

Заседание 24 сентября 2002 г. Г. вАн Дейк (Лейден). Обобщенные пары Гельфанда (обзор).

Группа $G=S L(2, \mathbb{R})$ действует на верхней комплексной полуплоскости дробно-линейными преобразованиями, причем пространство $L^{2}$ разлагается (без кратности) в прямой интеграл неприводимых представлений. Это свойство было изучено и обобщено Гельфандом и другими на пары $(G, K)$, где $G$ - группа Ли, а $K$ - компактная подгруппа. Аналогом верхней полуплоскости служит пространство $G / K$. Пары $(G, K)$, для которых $L^{2}(G / K)$ разлагается без кратности, называются парами Гельфанда. Наиболее известные примеры получаются, если $G$ - полупростая группа Ли, а $K$ - максимальная компактная подгруппа. Обсуждено обобщение понятия пары Гельфанда на ситуацию, когда $K$ - замкнутая, не обязательно компактная подгруппа $G$, и приведен ряд примеров.

Заседание 8 октября 2002 г. Встреча с представителем издательства "Мир" Г. М. Цукерман. Обсуждение плана издания математической литературы.

Заседание 15 октября 2002 г. Д. Сирсма (Нидерланды). Полиномиальные функции и их поведение на бесконечности.

По работе, выполненной совместно с М. Тибаром.

29 октября 2002 г. Совместное заседание Общества и Секции математики Дома Ученых.

К 200-летию со дня рождения выдающегося норвежского математика Нильса Хенрика Абеля.

Н. С. ЕрмолАЕВА. Жизнь и творчество Абеля.

А. В. ЯковлЕв. Теорема Абеля об алгебраических уравнениях.

В. А. МАлышев (Рыбинск). Интегралы с квадратическими иррациональностями.

М. А. СЕменов-Тян-ШАНСкий. Абелевы многообразия и интегрируемые задачи.

К. В. МАнуйлов. Теорема Абеля об аддитивных свойствах абелевых интегралов и функций.

5 ноября 2002 г. Совместное заседание Общества и Секции математики Дома Ученых.

С. К. ЛАндо (Москва). Инварианты узлов и инварианты графов.

Есть два принципиально различных способа сопоставить узлу в трехмерном пространстве граф̆. Первый из них состоит в том, чтобы рассмотреть проекцию узла на плоскость вдоль общего направления. В результате мы получаем регулярньй граф на плоскости, все вершины которого имеют валентность 4, причем в каждой вершине выделены "проходная" и "переходная"

Предыдущий отчет о работе Санкт-Петербургского математического общества см. в УМН, т. 57 , вып. 4 . 
пары противолежащих ребер. Второй подход принадлежит Васильеву и ассоциирует регулярный граф̆ специального вида (хордовую диаграмму) с "особым" узлом, имеющим простые самопересечения. Хордовую диаграмму можно сопоставить и плоской проекции узла. Инварианты хордовых диаграмм, которые приводят к инвариантам узлов, должны удовлетворять определенным ограничениям. Эти ограничения эффективно переносятся на “графы пересечений” хордовых диаграмм, которые, по сути, произвольны. Пространство, натянутое на произвольные графы, наделено естественной структурой алгебры Хопфа, а накладьваемые ограничения уважают эту структуру, что позволяет ввести на интересующем нас факторпространстве структуру факторалгебры Хопфа. В докладе рассказано о некоторых - весьма нетривиальных и далеких от ясного понимания - соотношениях между упомянутыми понятиями, приведено болшшое количество примеров инвариантов графов, порождающих инварианты узлов, а также высказаны некоторые гипотезы.

Заседание 18 февраля 2003 г. С. Ю. Пилюгин. Орбитальное отслеживание.

Пусть $f$ - гомеоморфизм метрического пространства $(X, \operatorname{dist})$. Фиксируем положительное число $d$. Последовательность $y=y_{k}$ называется $d$-nсевдотраекторией гомеоморфизма $f$, если выполнены неравенства $\operatorname{dist}\left(f\left(y_{k}\right), y_{k+1}\right)<d$. Гомеоморфизм $f$ обладает стандартньм свойством отслеживания, если по любому $\varepsilon>0$ можно указать такое $d>0$, что для любой $d$-псевдотраектории $y$ найдется точка $x$, удовлетворяющая неравенствам $\operatorname{dist}\left(f^{k}(x), y_{k}\right)<\varepsilon$. В докладе рассказано о введенных недавно орбитальных свойствах отслеживания, в которых вместо выполнения этих неравенств требуется выполнение либо включений $y \subset N(\varepsilon, O(x, f))$ или $O(x, f) \subset N(\varepsilon, y)$, либо неравенства $\operatorname{dist}_{H}(\operatorname{clos}(y), \cos (O(x, f)))<\varepsilon$, где $N(a, A)$ - $a$-окрестность множества $A, O(x, f)$ - траектория точки $x$ в динамической системе, порождаемой гомеоморфизмом $f$, a dist ${ }_{H}$ - метрика Хаусдорфа. Рассказано также о некоторых (иногда весьма неожиданных) связях введенных свойств с глобальной качественной теорией динамических систем.

25 марта 2003 г. Совместное заседание Общества и Общего семинара ПОМИ.

Э. А. Гирш. Полуалгебраические доказательства.

Сложность доказательств для логики высказьваний - активно развивающаяся область математики. Наличие доказательств, ограниченных по длине полиномом от размера доказываемого утверждения, означало бы равенство сложностных классов NP и соNP. Известны же лишь нижние (и верхние) оценки сложности доказательств для конкретных систем доказательств (и конкретных тавтологий, соответственно).

Первая часть доклада представляла собой введение в эту область и обзор известных систем доказательств и результатов о них.

Вторая часть доклада была посвящена результатам докладчика (совместным с Д. Ю. Григорьевым и Д. В. Пасечником), касающимся полуалгебраических (т.е. использующих рассуждения о полиномиальных неравенствах) доказательств. Например, вот доказательство принципа Дирихле:

$$
\begin{gathered}
\sum_{k=1}^{m}\left(\sum_{l=1}^{m-1} x_{k l}-1\right)+\sum_{l=1}^{m-1}\left(\sum_{k=1}^{m}\left(\sum_{k \neq k^{\prime}=1}^{m}\left(1-x_{k l}-x_{k^{\prime} l}\right) x_{k l}+\left(x_{k l}^{2}-x_{k l}\right)(m-2)\right)\right. \\
\left.+\left(\sum_{k=1}^{m} x_{k l}-1\right)^{2}\right)=-1 .
\end{gathered}
$$

(В докладе было сказано, почему.) Доказательства же этого принципа во многих других системах имеют экспоненциальную (от количества кроликов $m$ ) длину.

8 апреля 2003 г. Совместное заседание Общества и Секции математики Дома Ученых.

Актуальные проблемы школьного математического образования.

М. И. БАШмАков. Проект национального стандарта школьного математического образования.

А. Л. СЕменов (МИПКРО). Стандарты по математике и информатике в общем контексте российского школьного образования.

В. А. Рыжик. Цели школьного математического образования.

М. Я. ПРАТУСЕВИч. О содержании профильного образования.

Дискуссия. 
Как и на предыдущем заседании 23 апреля 2002 г. на ту же тему (см. УМН, т. 57, вып. 4, а также "Известия" за 31.05.2002), заседание приняло следующую резолюцию.

\section{О модернизации школьного математического образования.}

Санкт-Петербургское математическое общество видит необходимость совершенствования школьного математического образования, призывает членов общества активно участвовать в обсуждении путей его улучшения, готово внести свой вклад в решение проблем, возникающих в ходе модернизации всей системы образования. Общество разделяет обеспокоенность математической общественности Санкт-Петербурга происходящими изменениями в постановке школьного математического образования и озабочено необходимостью сохранения традиций высокого уровня математического образования, его роли и места в общем образовании, доступности для представителей всех слоев общества.

Общество принимает следующее решение.

1. Одобрить деятельность школьной комиссии по ряду важных направлений работы в школе - участие в подготовке стандарта школьного математического образования, проведение массовой игрь-конкурса "Кенгуру" (577 тыссяч участников в марте 2003 года), поддержка в создании членами общества школьных учебников, методическая работа с учителями.

2. Отметить снижение внимания к работе физико-математических школ города, утрату ряда завоеванньх позиций (в частности, отмену традиционной формы выпускного әкзамена, ослабление связей с математико-механическим факультетом Университета).

3. Поручить школьной комиссии направить в Министерство образования от имени Санкт-Петербургского математического общества письмо, в котором вьразить

- обеспокоенность математической общественности Санкт-Петербурга происходящими изменениями в постановке школьного математического образования;

- необходимость сохранения традиций высокого уровня математического образования, его роли и места в общем образовании, его доступности для представителей всех слоев общества;

- недопустимость принятия важных решений (утверждение стандарта образования, введение ЕГЭ, ограничение числа выпускаемых учебников, структура обучения в старшей школе) без предварительного и своевременного обсуждения математической и педагогической общественностью;

- готовность членов общества внести конструктивный вклад в решение проблем модернизачии образования.

4. Ходатайствовать перед Министерством образования о создании в Санкт-Петербурге регионального әкспертного совета по математике.

Президент Санкт-Петербургского математического общества проф. А. М. Вериик Председатель икольной комиссии общества академик РАО М. И. Башмаков

Заседание 15 апреля 2003 г. ЛорАн ЛАфОРГ (IHES, Франция, лауреат Филдсовской премии 2002 г.). Покрытия многогранников, склеивание клеток Шуберта и компактификация конфигурационньхх пространств.

Доклад представляет собой лекцию по проективной геометрии. При изучении компактификаций введенных Дринфелшдом пространств модулей “штук” с уровневой структурой или (по Фалтингсу) локалшных модулей многообразий Шимуры возникает задача о том, как компактифицировать факторы $P G L(r) \times \cdots \times P G L(r) / P G L(r)$ эквивариантным образом. Предлагается общий метод такой компактификации. Он также применим в случае конфигурационных пространств матроидов. Все получающиеся таким образом компактифицированные схемы снабжены структурньм морфизмом (который является гладким в случаях, когда факторов не более трех или когда ранг равен двум, но не в общем случае) над "торическим пучком", точки которого являются покрытиями некоторого целочисленного многогранника. Имеется индуцированная стратификация, слои которой могут быть описаны в терминах склеивания тонких клеток Шуберта. 
Все компактифицированные схемы имеют по крайней мере две модулярные интерпретации:

- как классификация эквивариантных векторных расслоений на некоторых торических многообразиях;

- как классификация определенных проективных рациональных многообразий с логариффмическими особенностями (которые обобщают "минимальные модели проективных пространств", введенные Фалтингсом).

Заседание 27 мая 2003 г. В. А. Тиморин (Москва). Алгебра, построенная по многочлену объема простого многогранника.

По всякому многочлену можно построить алгебру, профакторизовав алгебру дифференциальных операторов с постоянными коэффициентами по идеалу, аннулирующему данный многочлен. В докладе сделан обзор методов и результатов элементарной теории простых многогранников, связанных с алгеброй, построенной по многочлену объема. Определение этой алгебры принадлежит Пухликову и Хованскому. Алгебра моделирует кольцо когомологий гладкого проективного торического многообразия. Многие теоремы алгебраической геометрии (включая теорему Римана-Роха, сильную теорему Лефшеца, билинейнье соотношения Ходжа-Римана) имеют аналоги в элементарной геометрии простых многогранников и наиболее естественно формулируются в терминах многочлена объема.

Заседание 24 июня 2003 г. Я. М. ЭлиАшБЕРГ (Станфордский университет, США). Введение в симплектическую топологию: от теоремы Ролля до гомологий Флоера.

Возникшая около 20 лет назад симплектическая топология оказалась сегодня связанной со многими областями математики и теоретической физики: от гамильтоновой динамики, топологии трех- и четырехмерных многообразий, алгебраической геометрии до теории интегрируемых систем и теории струн.

В докладе были обсуждены основные идеи симплектической топологии и некоторые ее приложения.

Заседание 9 сентября 2003 г. И. Б. ФЕСЕнко (Ноттингем). Некоммутативная геометрия, нестандартная математика и теория эллиптических кривых с "вещественным умножением".

В последние годы появился ряд работ, в которых техника так назьваемой некоммутативной геометрии применяется к изучению (коммутативных) теоретико-числовых структур: например, работа А. Конна по дзета-функции Римана, работы Ю. И. Манина и М. Марколли по аракеловской геометрии, модулярным символам и модулярным формам, работа Манина по гипотетической теории "вещественного умножения" как некоммутативной версии классической теории эллиптических кривых с комплексным умножением.

В докладе были объяснены некоторые из соответствующих структур и основных идей, а затем предложен новьй, более универсальный, подход, который работает не только на уровне алгебраических структур, но и на уровне целостных арифметических структур.

Этот подход основьвается на принципе гипердискретизации из нестандартной математики и его многочисленных приложениях. В ряде случаев теневой образ гиперкоммутативной конструкции должен быть тесно связан с некоммутативным описанием посредством обобщения известного в теории струн отображения Зайберга-Виттена.

Заседание 7 октября 2003 г. В. А. ВАСИльЕВ (Москва). Когомологии пространства узлов и их комбинаторные формулы.

Теория инвариантов узлов является лишь частью более естественной задачи вьгисления кольца когомологий пространства узлов в $\mathbb{R}^{n}, n \geq 3$. Любой такой класс когомологий (например, инвариант) можно задать индексом пересечения с подходящим классом относительных гомологий в пространстве узлов. Комбинаторной формулой для него назьвают простой полуалгебраический цикл, реализующий этот класс гомологий. Наиболее известный пример комбинаторных формул для инвариантов - это диаграммы Поляка-Виро.

В докладе рассказано о вычислении старших классов когомологий и описан эффективный (т.е. не требующий моделирования непрерывных процессов, деформаций пространственных объектов, ray-tracing и пр.) комбинаторньй алгоритм для нахождения комбинаторных формул (в том числе и для инвариантов). Этот алгоритм основан на аналогии теории узлов с комбинаторной теорией наборов афффинных плоскостей и часто является простейшим или единственньм доказательством существования класса когомологий.

Заседание 21 октября 2003 г. Г. Ю. ПАнинА. Гиперболические виртуальные многогранники и гипотеза единственности в теории вьшуклых поверхностей. 
Рассказаны и обсуждены контрпримеры к старой гипотезе: если радиусы главной кривизны поверхности гладкого трехмерного тела $K$ всюду разделены постоянной $C$, то $K$ есть шар радиyса $C$.

Доклад основан на работах А. В. Погорелова, И. Мартинеса-Мора и докладчика.

25 ноября 2003 г. Совместное заседание Общества и Секции математики Дома Ученых. A. Н. Колмогоров, Дж. фон Нейман - математические гении XX века. К столетию выдаюшихся ученых.

С докладами выступили: В. М. Тихомиров (Москва), А. М. ВЕРшик. Были заслушаны выступления М. А. СеменовА-Тян-ШАнСкоГО, В. СерГеЕвА (Москва), М. А. КраСнопеРОвой.

Санкт-Петербургское отделение Математического института им. В. А. Стеклова РАН, Международный математический институт им. Эйлера при участии Санкт-Петербургского математического общества.

Конференция памяти Л. В. Канторовича (1912-1986)

“Математика и Экономика: Старые проблемы и новые подходы" 8-13 января 2004 г.

Пленарные заседания.

В. Л. МАКАРОв (Москва). Оптимизация по Канторовичу и исчисление институтов.

В. М. ПолТЕРович (Москва). Инновации, имитация и экономическое развитие.

Б. Т. Поляк (Москва). Метод Ньютона-Канторовича и его глобальная сходимость.

Г. Ю. ТрОфимов (Москва). Л. В. Канторович и теория экономического роста.

Л. КлЕйн (Филадельфия). Оценки продуктивности информационных технологий с точки зрения затрат - вьшуска за последние три десятилетия на примере США.

К. Шмидт (Париж). Линейное программирование, теория игр: исторический обзор и прогнозы на будущее.

М. ДЕмпСтЕР (Кембридж, Великобритания). Стохастическая динамическая оптимизация.

Дж. ГУмеРмАН (Санта Фе, США). Адаптация и интенсификация сельского хозяйства доисторического американского юго-запада.

Секция "Проблема Монжа-Канторовича".

А. М. ВеРшик (С.-Петербург). К истории метрики Канторовича и ее приложений в динамических системах.

В. ГАнгьо (Атланта). Приложения проблемы Монжа-Канторовича к кинетической теории,

А. ШниреЛЬмАн (Великобритания). Проблема Монжа-Канторовича и гидродинамика.

В. Л. ЛЕвин (Москва). Проблема Монжа-Канторовича: точные решения и приложения к теории принятия решений.

А. Н. СоБОлЕвСКий (Москва). Оптимальные методы перемещения масс в космологии.

И. ЯрошевСКА (Краков). Стабильность мер и решений дифференциальных уравнений и принцип максимума Канторовича-Рубинштейна.

Секция "Физическая экономика".

В. М. СЕРГЕЕВ (Москва). Термодинамический подход к рыночному равновесию, рациональность, права собственности.

Д. Смит (Санта Фе, США). Классическая термодинамика и экономическая теория общего равновесия.

Д. ЛЕйтЕс (Стокгольм). Параметры стабильности неголономных систем (от супергравитации до рыночной экономики).

А. С. Кузьмин (Москва). Классификация инвестиционных механизмов и математическое моделирование инвестиционных рынков.

Р. Г. ХлЕБопРОс (Красноярск). Почему люди любят и ненавидят капитализм, и почему социализм невозможно уничтожить. 
Секция "Математико-экономические модели".

В. А. ВАСИльЕВ (Новосибирск). Онекоторых К-пространствах бесконечных кооперативных игр.

В. И. Аркин (Москва). Инвестирование в условиях неопределенности, налогообложение и проблема оптимальной остановки.

В. И. ДАнилов (Москва). Дискретная вьшуклость на целочисленной решетке.

А. А. ШАНАНин (Москва). Проблема интегрируемости в теории рационального поведения.

В. Д. МАТвеЕнко (С.-Петербург). Теория экономической динамики российской экономики.

В. М. МАРАКУлин (Новосибирск). Анализ равновесия в пространствах Канторовича.

Э.А. МухачевА (Уфа). Л.В. Канторович и задачи раскроя-упаковки: 50 лет становления и развития.

С. Л. ПЕчЕРский (С.-Петербург). Некоторые приложения теории суперлинейных многозначных отображений к теории игр.

С. М. МЕньшиков (Амстердам). Модель Канторовича - актуальность в наши дни.

Секция "Функциональный анализ".

Г. Л. Литвинов (Москва). Деквантизация и идемпотентный функциональный анализ.

А. ИоффЕ (Хайфа). О теореме Хана-Банаха-Канторовича.

А. А. Флоринский (С.-Петербург). О значениях векторнозначных мер.

С. С. КУТАТЕЛАДЗЕ (Новосибирск). Путь и пространство Канторовича.

Мемориальное заседание, посвященное Л. В. Канторовичу.

С. П. Новиков, Л. Д. ФАДДЕЕВ, Э. Б. ЕРШОВ, А. М. ВЕРШик, Н. А. ШАНИн и др.

24 февраля 2004 г. Заседание, посвященное памяти профессора Г.И. Натансона (1930-2003).

С воспоминаниями выступили: В.М. БАБИч, В.С. ВИДЕНСКИЙ, О.Л. ВИНОГРАДОВ, И.К. ДАУГАВЕТ, В.В. ЖУК, Б.М. МАКАРОВ, Я.Г. НАТАНСОН, И.В. НЕДЗВЕЦКАЯ, В. П. ОДИНЕЦ, А. Н. ПОДКОРЫТОВ, М. А. СКОПИНА, В. Л. ФАЙНШМИДТ, В. П. ХАВИН.

16 марта 2004 г. Заседание, посвященное памяти академика О. А. Ладыженской $(1922-2004)$.

С воспоминаниями выступили: Н. Н. УРАЛЬцЕВА, М.С. БИРман, Н. М. ИвОчКИНА, Г.А. СЕРЕГИН, А. Л. СКУБАЧЕвСКИй (Москва), А. В. ФУРСиков (Москва), Э. А. ТРОПП, А. М. ВЕРшик. Был показан видеофильм об О. А. Ладыженской.

\section{Математический лекторий для студентов}

5 ноября 2002 г. С. К. ЛАндо (Москва). Что такое тангенс.

28 ноября 2002 г. М. А. Лифшиц. “Звездная пыль" и вероятность.

19 сентября 2003 г. Ю. В. МАТиясЕВИЧ. Десятая проблема Гильберта: что можно и что нельзя делать с диофантовыми уравнениями.

25 марта 2004 г. С. К. ГОдунов (Новосибирск). О гарантированной точности в спектральных задачах.

\section{Премия "Молодому математику"}

Премии "Молодому математику" удостоены:

За 2002 год - А. Г. Эршлер за работу “Асимптотики характеристик случайных блужданий на разрешимых группах".

За 2003 год - А. Н. Зиновьев за работу "Обобщенные явные формулы Артина-Хассе и Ивасавы”. 